\title{
Parameters Determination of GTN Model and Damage Analysis of Aluminum Alloy 6016 Sheet Metal
}

\author{
Yu-Mei HU ${ }^{1,2}$, Min-Zhi CHEN ${ }^{1, a,{ }^{,}}$, Yue XIAO ${ }^{1}$, Jin XIAO ${ }^{1}, X$ TAN $^{1}$, Qi TANG ${ }^{1}$, \\ Ying-E ZHOU ${ }^{2}$, Tai-Song CUI ${ }^{2}$
}

${ }^{1}$ State Key Laboratory of Mechanical Transmission, Chongqing University, China

${ }^{2}$ State Key Laboratory of Vehicle NVH and Safety Technology, Chongqing401122, China

achenmz_jx@126.com

${ }^{*}$ Corresponding author

Keywords: GTN Model, Damage, AA6016.

\begin{abstract}
Taking the material of AA6016 (Aluminum Alloy 6016) which is widely used in automobiles as the object of this study, the mechanical properties based on GTN (Gurson-Tvergaard-Needleman) model had been investigated. The parameters of the GTN model which were used for simulation were identified by uni-axial tensile test, parameter fitting and the inverse finite element method. Then, the simulation of the uni-axial tensile test was done by developing subroutine of UMAT (User-Defined Material) based on LS-DYNA. The results show that the damage and fracture of AA6016 have good agreements with the experiment. Further, the influences of different damage parameters $f_{0}$ and $f_{n}$ on the mechanical properties were investigated, which verifies the rationality of the GTN parameters. It is of interest that in the results of both simulation and experiment, the direction of fracture on the plate tensile specimen is $60^{\circ}$, instead of the $45^{\circ}$ or $90^{\circ}$ obtained in previous literatures.
\end{abstract}

\section{Introduction}

AA6016 has been successfully used in automobile panels due to its excellent ductility in recent years. However, with the accumulation of the deformation during the process of sheetforming, there is a possibility that cracks and even fractures will occur, which will lead to a lot of waste and reduce the products pass rate. It is hard to avoid and has plagued the actual production for a long time.

The research of mesoscopic damage mechanics[1] has pointed out that for material such as AA6016 which without initial macro-cracks, the damage and fracture appeared during the process of sheet forming is mainly the result of the nucleating, growing and coalescing of micro-voids or micro-cracks. So the choice of a suitable material constitutive model with micro-cracks and micro-voids will contribute to understanding the damage and fracture mechanism of AA6016 in the process of sheet forming.

Since McClintock was first and foremost in establishing the micro-void material constitutive model[2], macro damage mechanics were combined with micro damage mechanics in such a way that it offered researchers a new method to solve the damaging and fracturing phenomenon of material without the initial macro cracks.

Based on the research of McClintock and Rice[4], Gurson[3] developed the well-known Gurson model. He abandoned the hypothesis of infinite body and developed a finite cell model containing micro voids, which laid the foundation for the description of damage(such as taking the void volume fraction as the damage variable), and the plastic theory of macroscopic volume expansion.

In 1979, Tvergaard and Needleman[5,6] modified the Gurson model by introducing parameters $q_{1}$ and $q_{2}$ and established the void fusion equation $f^{*}$, which further corresponds to the evolution process in electron microscopy experiment. The modified Gurson model is called GTN model. Though the GTN model has been widely accepted as a typical model used to study the material'sinner mesoscopic damage, the complication of numerical method and excessive damage parameters make it difficult to describe the evolution process of inner voids. 
In this paper, the uni-axial tensile test for AA6016 sheet metal was done, combined in combination with parameter fitting, to obtain parts of the mechanical parameters of the GTN model. Then the simulation of uni-axial tensile was done by developing subroutine of UMAT (User-Defined Material)) based on LS-DYNA, and the left unknown parameters of the GTN model were obtained by using inverse finite element method [16]. Additionally, the influences of $f_{0}$ and $f_{n}$ on evolution process of voids are also discussed.

\section{The GTN Model}

\section{Modified GTN model}

The plastic potential state equation of GTN model is shown as follows:

$$
\varphi=\left(\frac{\sigma_{e q}}{\bar{\sigma}_{m i s}}\right)+2 q_{1} f^{*} \cosh \left(-\frac{3 q_{2} \sigma_{H}}{2 \bar{\sigma}_{m i s}}\right)-\left(1-f^{* 2}\right) .
$$

where $\sigma_{e q}=\sqrt{3 / 2 S_{i j} S_{i j}}$ is the macroscopic Von-Mises equivalent stress, $-\sigma_{H}=1 / 3 \sigma_{k k}$ is the macroscopic hydrostatic stress, $S_{i j}=\sigma_{i j}-1 / 3 \sigma_{k k} \delta_{i j}$ is the deviatoric stress tensor, $\sigma_{i j}$ is the flow stress, $\delta_{i j}$ is the Kronecker delta, and $\bar{\sigma}_{m i s}$ is the equivalent stress of the matrix material; $q_{1}$ and $q_{2}$ are the correction coefficients introduced by Tvergaard and Needleman, with $q_{2}=q_{1}^{2} ; f$ is the void volume fraction, and $f^{*}$ is the function of $f$ and it is defined as follows:

$$
f^{*}=\left\{\begin{array}{c}
f \\
f_{c}+k k\left(f-f_{c}\right)
\end{array} .\right.
$$

where $f_{c}$ represents the critical value when the void coalescence occurs, $k k$ is the void growth acceleration factor corresponds to porosity rate after voids grew and is shown as follows:

$$
k k=\frac{f_{n}^{*}-f_{c}}{f_{f}-f_{c}} .
$$

where $f_{n}^{*}$ is the maximum admissible void volume fraction when stress bearing capacity equals to zero, and $f_{f}$ is the critical value corresponds to the complete damage of the material.

From the Eq. 2 it is clearly that as $f \rightarrow f_{f}$ and $f^{*} \rightarrow f_{n}^{*}$, the material will lose its bearing capacity gradually. To ensure $\boldsymbol{\sigma}_{i j}=\mathbf{0}$ when it comes to the complete damage of the material, $f_{n}^{*}=1 / \mathrm{q}_{1}$ can then be obtained from Eq. 1 and Eq. 2. And when $f^{*}=f$ and $f^{*}=0$, Eq. 1 degrades into Gurson model and Von-Mises yield function respectively.

In porous materials, the plastic flow potential $\varphi$ not only relates to void volume fraction $f$, but also depends on $\bar{\varepsilon}_{m i s}^{p l}$, which represents the cumulative plastic strain under material micro state and can be obtained by equivalent plastic power principle as follows:

$$
\begin{aligned}
& (1-f) \bar{\sigma}_{m i s} d \bar{\varepsilon}_{m i s}^{p l}=\boldsymbol{\sigma}: d \boldsymbol{\varepsilon}^{p} . \\
& d \bar{\varepsilon}_{m i s}^{p l}=\frac{\boldsymbol{\sigma}: d \boldsymbol{\varepsilon}^{p}}{(1-f) \bar{\sigma}_{m i s}} .
\end{aligned}
$$

where $d \bar{\varepsilon}_{m i s}^{p l}$ is the cumulative equivalent plastic strain increment of the matrix material, and $d \boldsymbol{\varepsilon}^{p}$ represents the macroscopic plastic strain increment. 


\section{Void Volume Fraction $\boldsymbol{f}$ of Voids Evolution GTN Model}

The modified GTN model involved the evolution equation of void volume fraction.It divides the damage evolution into two parts, which describes the growth of existing voids and nucleation of new voids. In this way the void volume fraction $f$ will be influenced. The function is characterized by:

$$
d f=d f_{\text {growth }}+d f_{\text {nucleartion }}
$$

where $f_{\text {growth }}$ denotes the growth of voids, while $f_{\text {nucleartion }}$ describes the contribution of newly nucleated voids.

As the material is incompressible, the existing void volume fraction $f_{\text {growth }}$ is related to mean plastic strain and can be written as:

$$
d f_{\text {growth }}=(1-f) d \boldsymbol{\varepsilon}^{p}: \boldsymbol{I} .
$$

here, $\boldsymbol{I}$ is the third order unit tensor and $\mathrm{d} \varepsilon^{\mathrm{p}}$ stands for the plastic strain rate.

The nucleation of voids is described by:

$$
d f_{\text {nucleartion }}=A_{N} d \bar{\varepsilon}_{\text {mis }}^{p l} \text {. }
$$

The void nucleation constant is shown as follow:

$$
A_{N}=\frac{f_{n}}{s_{N} \sqrt{2 \pi}} \exp \left\{-\frac{1}{2}\left(\frac{\bar{\varepsilon}_{m i s}^{p l}-\varepsilon_{N}}{s_{N}}\right)^{2}\right\} .
$$

here, $A_{N}$ is the constant represent the void nucleation, and $f_{n}$ is the volume fraction of void nucleating particles. $A_{N} / f_{n}$ follows the normal distribution, in which $\varepsilon_{N}$ and $\mathrm{s}_{\mathrm{N}}$ are the mean strain (expectation in mathematics) and the corresponding standard deviation respectivelyboth for void nucleation. In conjunction with Eq. 7, 8, the evolution equation of voids can be obtained in the form:

$$
d f=(1-f) d \boldsymbol{\varepsilon}^{p}: \mathbf{I}+\frac{f_{n}}{s_{N} \sqrt{2 \pi}} \exp \left\{-\frac{1}{2}\left(\frac{\bar{\varepsilon}_{m i s}^{-p l}-\varepsilon_{N}}{s_{N}}\right)^{2}\right\} d \bar{\varepsilon}_{m i s}^{-p l} .
$$

Based on the experimental investigation, Tvergaard and Needleman have concluded the recommended material constants: $\varepsilon_{N}=0.1, s_{N}=0.3$.

Above all, GTN model include many unknown parameters, including elastic modulus E, Poisson's ratio $v$, yield point stress $\sigma_{y}$ and damage parameters $f_{0}, f_{c}, f_{n}, f_{f}, q_{1}, q_{2}$. This paper would get those parameters by means of experiment and inverse finite element method.

A complete implicit Euler strain update algorithm [7] is applied to solve the numerical simulation of GTN model. Aravas [8] and Zhang [9] have proved it by numerical analysis. This paper uses LS-DYNA software and customs probability damage constitutive model, combining UMAT subroutine, to analysis the GTN model and confirm the damage parameters. In addition,the UMAT assumes that $\bar{\varepsilon}_{m i s}^{p l}=h s v(1)$ is the accumulated plastic strain of matrix material, while $f=h s v(2)$ is the accumulated void volume fraction[10].

\section{Simulation of Sample Plate}

\section{Uniaxial Tensile Experiment}

Physical dimensions of the specimen of AA6016 are shown in Fig.1. The specimen was stretched with a speed of $1.0 \mathrm{~mm} / \mathrm{min}$ in the uni-axial tensile test and the obtained fracture morphology shows that necking phenomenon didn't occur, which can be seen in Fig. 2. So it's reasonable for considering the engineering stress-strain curve as the real stress-strain curve. 


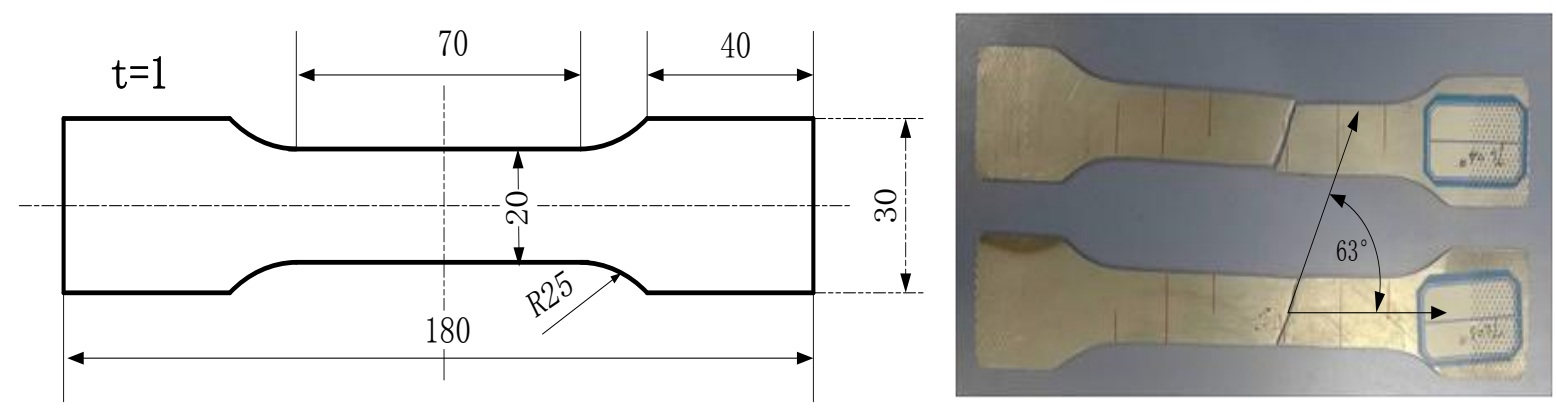

Fig.1 Plate tension specimen

Fig.2 Fracture morphology ofplate tensile specimen

Provided that the void volume fraction $f$ doesn't have any effect on AA6016, the equivalent stress versus equivalent plastic strain response $[11,12]$ is represented through the following form:

$$
\frac{\sigma}{\sigma_{y}}=\left(\frac{\sigma}{\sigma_{y}}+\frac{3 G}{\sigma_{y}} \varepsilon^{p}\right)^{N} .
$$

in this formula, $\sigma_{y}$ and $\sigma$ correspond to the yield stress and the equivalent stress respectively, $\varepsilon^{p}$ represents the plastic strain, $\mathrm{G}$ is the shear modulus, and $\mathrm{N}$ is the plastic hardening exponent.

Considering the material's stress will increase with the strain without the influence of porosity, fitting Eq. (11) would only depend on the first half part of the experimental data 1 or data 2 which are shown in Fig.3.The fitting results are: the material elastic modulus $E=61674.38 \mathrm{MPa}$, the Poisson's ratio $v=0.3$, the plastic hardening exponent $N=0.13$, and the yield stress $\sigma_{y}=$ 137.3 MPa.

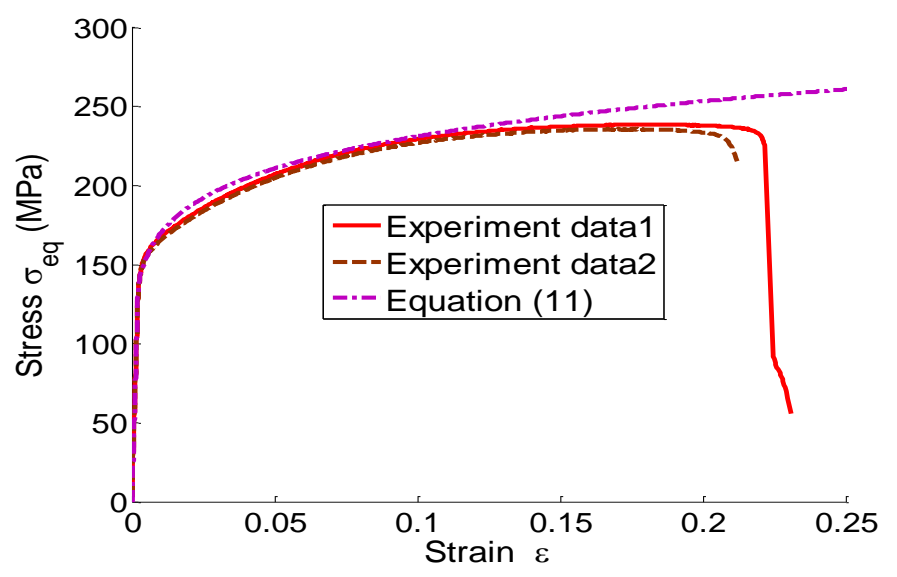

Fig.3 Stress-strain curves of plate tensile specimens

\section{GTN Damage Parameters Identification}

On account of AA6016 panel is plastic (extensible) material and its ductile fracture is mainly caused by the growth of voids, it is reasonable to assume the void fusion factor $k k=1[13,14]$. According to the analysis given by G. Vadillo.et.al. [15], the hypothesis of damage parametersis made to: $q_{2}=0.8, \varepsilon_{N}=0.1, s_{N}=0.3$, GTN parameters such as $f_{0}, f_{n}, f_{f}$, are obtained from the inverse finite element method[16], and all the parameters are listed in Table 1.

Tab. 1. Parameters of GTN constitutive model.

\begin{tabular}{cccccc}
\hline \multirow{4}{*}{ AA6016 } & $E$ & $v$ & $\sigma_{y}$ & $q_{1}$ & $q_{2}$ \\
\cline { 2 - 6 } & 6167.38 & 0.3 & 137.3 & 1.88 & 0.8 \\
\cline { 2 - 6 } & $\varepsilon_{N}$ & $s_{N}$ & $f_{n}$ & $f_{0}$ & $f_{f}$ \\
\cline { 2 - 6 } & 0.3 & 0.1 & 0.15 & 0.001 & 0.031 \\
\hline
\end{tabular}


The FEM model and boundary condition of AA6016 panelare shown in Fig. 4. Fig.5 shows the distribution contour of the void volume fraction $f(h s v(2))$ that got from the simulation.

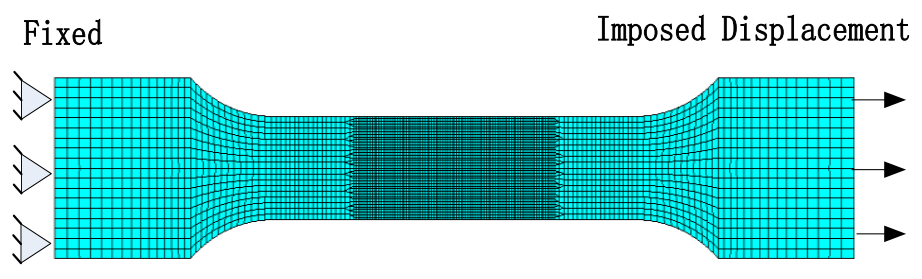

Fig. 4 Boundary Conditions of plate tension specimen

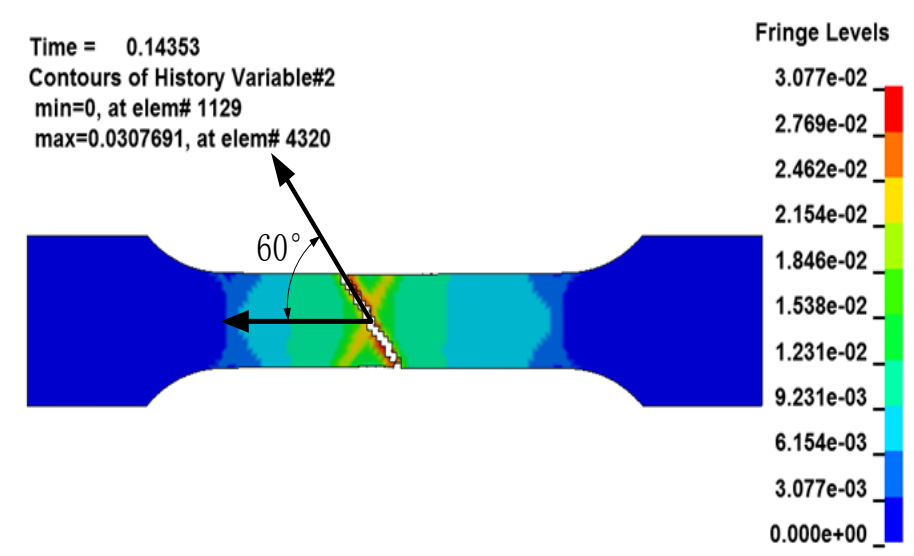

Fig. 5 Void volume fraction $f(h s v(2))$ of plate tension specimen

It can be seen that the void volume fraction $f$ mainly focus on the surface of the fracture when the plate specimen was broken. The fracture surface versus the DX tensile direction is $60^{\circ}$ in FEM simulation(Fig.5), while it is $63^{\circ}$ in the experimental result (Fig.2). Thus, the fracture appeared in simulation result is similar to the experimental result. However, previous literatures show that the fracture surface versus the DX tensile direction is $45^{\circ}$ or $90^{\circ}$, which is not the same with our results.

The tensile stress versus tensile strain relationship of AA6016 are shown in Fig. 6, where '--' represents the pure plastic stress-strain curve (Eq.11), '- ' is the experimental result, and 'o' is the simulation result obtained from the GTN model. It is clear that with the increment of plastic strain $\varepsilon^{p}$, the void volume fraction $f$ imposes an increasing influence on the mechanical behavior of AA6016, and when $f$ comes to 3.1e-2 (see Fig.5, Table 1), the failure would occurs. Fig.6 has shown that the curves of the GTN model and the experiment are very similar. Therefore, it indicates that the parameters of the GTN model are applicable for AA6016.

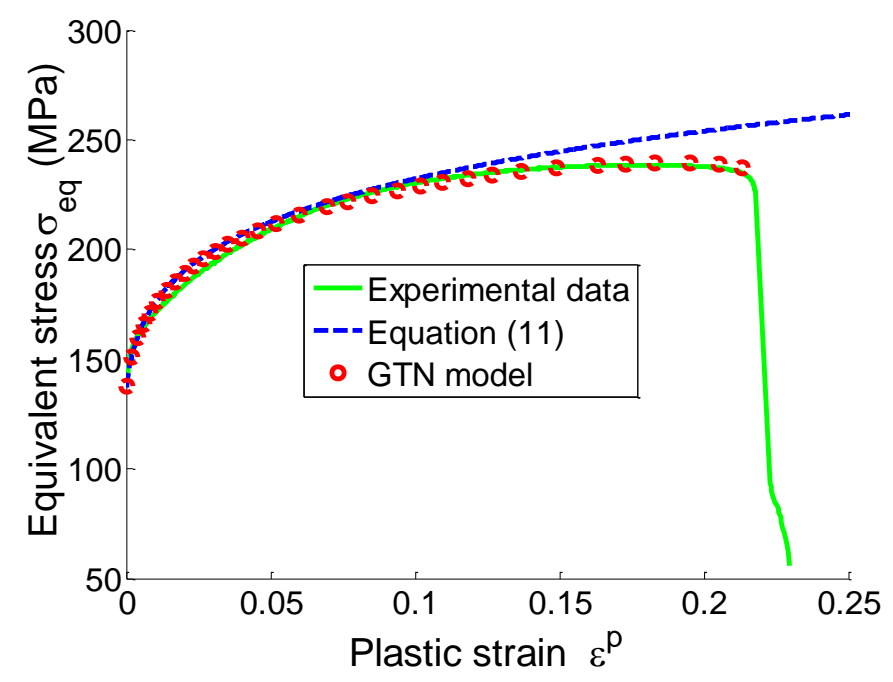

Fig. 6 Contrast of equivalent plastic stress-strain curves 


\section{Influence of GTN Damage Parameters on $f$}

The plastic potential state equation Eq. 1 shows that the variation condition of macro equivalent stress in evolution process is similar to that condition of $f$ versus the plastic strain. According to the basic theory of GTN model, the propagation of void volume fraction $f$ includes three main aspects as following:

Firstly, from Eq. 1, it can be seen that when $f \geq f_{c}$, the void growth acceleration factor $k k$ has great effects on void coalescing. Owing to the previous assumption that $k k=1.0$, the effect of $f_{c}$ on the damage of AA6016 is not considered.

Secondly, Eq. 10 is the iterative equation of void volume fraction $d f$, including void nucleation and growth. Due to the relationship of $f_{n}$ and $A_{N}$ as shown in Eq. 9, $f_{n}$ has an influence on $A_{N}$. And Fig.7 shows the influence of different values of $f_{n}$ on the equivalent stress-plastic strain curve. With the growth of $f_{n}$, the simulated GTN curve will gradually deviate from the elastic-plastic curve given by Eq. 6 . While $f_{n}=0.16$, the simulated GTN curve and the experimental curve can match together very well. Furthermore, when $f_{n}>0.16$, the simulated GTN curve will gradually deviate from the experimental one. Another phenomenon is shown in Fig.8, when $f_{n}=0$, that is the void volume fraction $f$ is nearly 0 , and the rate of $f$ versus $f_{n}$ increases, which will deviate the GTN simulated curve from the elastic-plastic one.

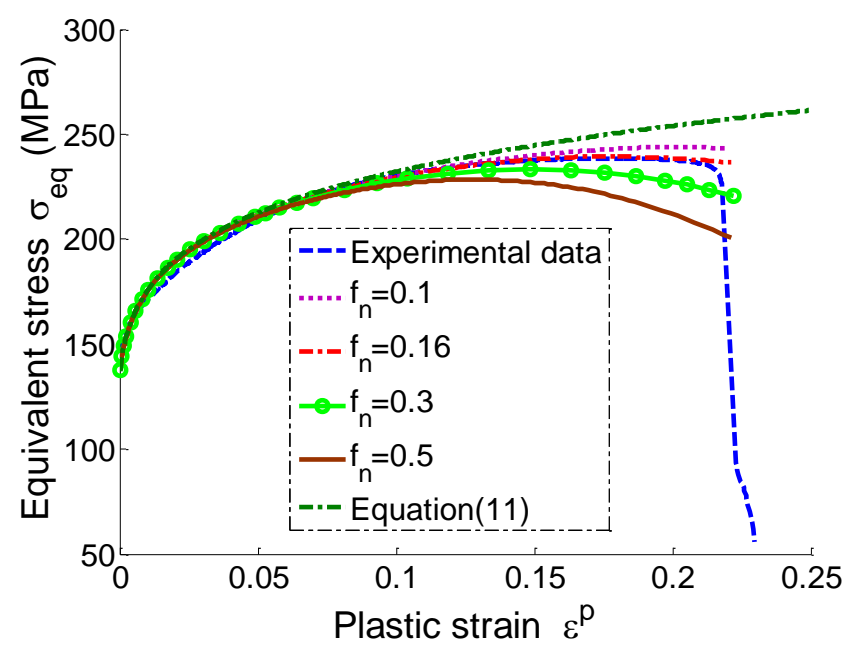

Fig.7 Equivalent stress-plastic strain curves for different $f_{n}$ parameters

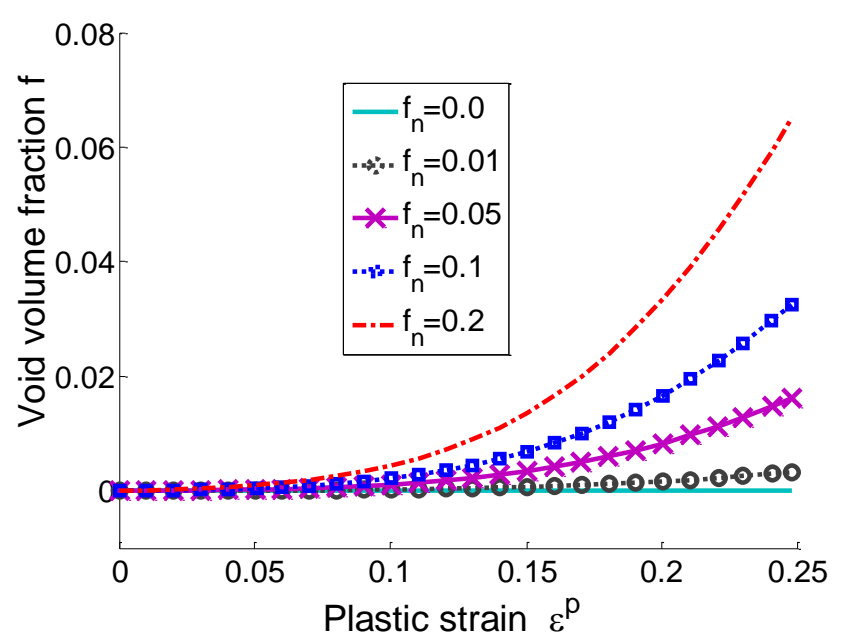

Fig.8 Void volume fraction-plastic strain curves for different $f_{n}$ parameters

Thirdly, that the initial void volume fraction $f_{0}$ is used to depict the voids produced in the manufacturing of roughcast which has an effect on the damage of ductile material in a certain range. 
$\operatorname{Kim}[17]$ assumed that $f_{0}$ ranges from 0.001 to 0.025 for the common material. Therefore,simulations were done by setting the value of $f_{0}$ from 0.001 to 0.03 to study the effect on stress-strain curve of AA6016 sheet metal. When $f_{0}$ changes in the range of 0 to 0.001 , the material mechanical properties will be less affected as shown in Fig.9. While $f_{0}>0.01$, the differences between the experimental curve and the plastic strain curve calculated by simulation will become bigger, which means the effect of $f_{0}$ on material stress-strain curve will become bigger as well, and further affects the stamping process of the material. As a result, problems such as crack initiation and nuclear cracking will appear.

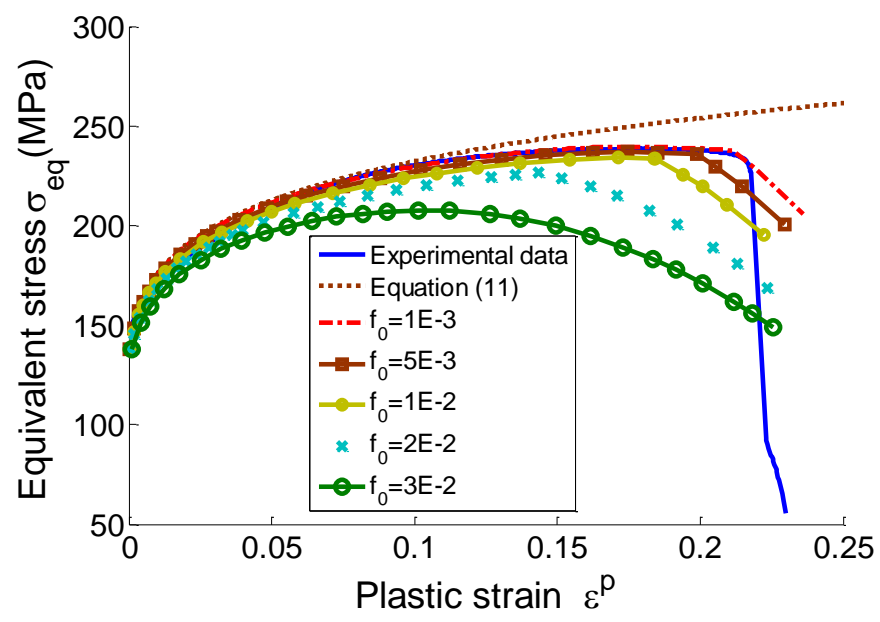

Fig.9 Equivalent stress-plastic strain curves for different $f_{0}$ parameters

\section{Results and Discussion}

By experiment and inverse finite element method, the parameters of the GTN model for AA6016 sheet metal were finally identified. Meanwhile, the influences of damage parameters $f_{0}$ and $f_{n}$ on the voids evolution progress and the fracture progress were alsodiscussed in this paper. The conclusions are shown as follows:

1)The influence of the $f_{n}$ in the GTN model on voids evolution shows that it has a distinct effect on fitting the stress-strain curve of AA6016. Though it is widely acknowledged that the GTN model's damage parameter $f_{n}$ ranges from 0.01 to 0.06 , in this investigation, the results of simulation and experiment were in agreement while $f_{n}=0.16$. The reason may be that the AA6016 is a super plastic material and the failure strain could reach to 0.21, so that the value of AA6016's damage parameter $f_{n}$ exceeds the general numeric value.From this aspect it shows that the nucleation coefficient $f_{n}$ will contribute to the void's evolution progress.

2) Through the research on initial void volume fraction $f_{0}$, it is obvious that the initial voids have a wide influence on the mechanical properties of damage and fracture.Fig.9 shows that when $f_{0}$ varies from 0.00 to 0.001 , it has little influence on the mechanical properties, however, when $f_{0}$ exceeds 0.01, the influence becomes greater, and the simulation curve will deviate from the experimental curve more and more. It indicates that the research on $f_{0}$ will be helpful in inspecting whether the AA6016 blank is qualified.

\section{Acknowledgements}

The authors gratefully acknowledge the financial support from the State key Laboratory of Vehicle NVH and Safety Technology.

\section{References}

[1] YuShouwen, Damage micro mechanic, Tsinghua University Press, Beijing, 1997. 
[2] F.A. McClintock, A criterion for ductile fracture by the growth of holes, Appl. Mech. (1968) $363-371$.

[3] A.L. Gurson, Continuum theory of ductile rupture by void nucleation and growth: Part-I: Yield criteria and flow rules for porous ductile media, Trans. ASME J. Eng. Mater. Technol.99 (1977) $2-15$.

[4] J.R. Rice, D.M. Tracey, On the ductile enlargement of voids in triaxial stress fields, Mech. Phys. Solids. 17 (1969) 201-217.

[5] V. Tvergaard, A. Needleman, Analysis of the cup-cone fracture in a round tensile test bar, ActaMetallurgica. 32 (1984) 157-169.

[6] A. Needleman, V. Tvergaard, An analysis of ductile rupture in notched bars, Mechanics and Physics of Solids. 32 (1984) 461-490.

[7] JC. Simo, RL.Taylor, Consistent tangent operators for rate independent elastoplasticity, Computer Methods in Applied Mechanics and Engineering.48 (1985) 101-119.

[8]N.Aravas, On the numerical integration of a class of pressure-dependent plasticity models, International Journal for Numerical Methods in Engineering. 24 (1987) 1395-1416.

[9] ZL Zhang, Explicit consistent tangent moduli with a return mapping algorithm for pressure-dependent elastoplasticity models, Computer Methods in Applied Mechanics and Engineering. 121 (1995) 29-44.

[10] LS-Dyna:Keyword User's Manual Volume II Material Models.Version R7.0 2013[M]

[11] Kim Jinkook, GaoXiaosheng, Srivatsan, Modelling of void growth in ductile solids: effects of stress triaxiality and initial porosity, Engineering Fracture Mechanics. 71 (2004) 379-400.

[12] BonoraNicola, Gentile Domenico, Pirondi A, et al., Ductile damage evolution under triaxialstate of stress: theory and experiments, International Journal of Plasticity. 21 (2005) 981-1007.

[13] JinkookKim,XiaoShenggao, Modelling of void growth in ductile solid:effects of stress triaxiality and initialporosity, EngineeringFractureMechanics. 71 (2004) 379-400.

[14]Chen Zhiying, Comparison of GTN Damage Models for Sheet Metal Forming, J. Shanghai Jiaotong Univ. 13 (2008) 739-743.

[15]G. Vadillo, J. Fernández-Sáez, An analysis of Gurson model with parameters dependent on triaxiality based on unitary cells, European Journal of Mechanics. 28 (2009) 414-427.

[16]Huang Xi-cheng,ChenYuze, et al, Identification of Meso-damage Parameters of 2169 Steel, Journal of Materials Engineering. 4 (2007) 50-52.

[17] J.Kim, X.Gao, S.Srivatsan, Modelling of void growth in ductile solids: effects of stress triaxiality and initial porosity, Engineering Fracture Mechanics. 71 (2004) 379-400. 